\section{Associação entre sintomas de insônia, cochilo diurno e quedas em idosos da comunidade}

\author{
Association between insomnia symptoms, daytime \\ napping, and falls in community-dwelling elderly
}

\author{
Asociación entre síntomas de insomnio, siesta \\ breve diurna y caídas en ancianos de una \\ comunidad desfavorecida
}




\section{Introdução}

As quedas representam um grave problema de saúde pública com implicações diretas para a qualidade de vida e o bem-estar da população idosa 1,2,3. Elas têm elevado custo econômico e social, principalmente quando o idoso sofre diminuição da autonomia e da independência a ponto de necessitar de cuidados especializados em domicílio ou em instituições 4,5. Estima-se que $30 \%$ dos idosos que vivem na comunidade sofrem queda a cada ano, e destes, metade sofre quedas recorrentes 2 .

Semelhante a outras condições que afetam o idoso, tais como delirium e incontinência urinária, a queda é considerada uma síndrome geriátrica ${ }^{6}$. É caracterizada pela contribuição de múltiplos fatores de natureza intrínseca acompanhada ou não de riscos ambientais, e pela interação entre doenças crônicas predisponentes e eventos agudos precipitantes 1,2,6,7. Pesquisas têm revelado que a ocorrência de quedas é maior entre as mulheres, nos mais idosos, aqueles que apresentam declínio funcional em atividades de vida diária, em idosos com histórico de quedas e naqueles com alterações visuais, auditivas, na marcha e no equilíbrio 6,7,8,9,10,11,12. Doenças tais como depressão, hipertensão, insuficiência cardíaca, incontinência urinária, osteoporose e doença de Parkinson, bem como o maior número de doenças associadas configuram-se, igualmente, como fatores de risco para quedas em idosos $6,7,11,13$. O risco de quedas na população idosa se eleva com o aumento do número de medicações referidas para uso contínuo e conforme o consumo de medicamentos psicoativos 7,9,14. A ocorrência de quedas representa risco aumentado de morte entre idosos, de modo especial entre aqueles com idade avançada 2,15,16.

Problemas relacionados ao sono e a frequência e duração de cochilo diurno estão entre os fatores de risco de quedas em idosos da comunidade menos estudados 11,12,17,18,19,20,21,22, em comparação com outros tais como histórico de quedas, diminuição do equilíbrio e da força muscular, alteração na marcha, consumo de psicotrópicos, depressão e limitação funcional em atividades de vida diária 6,7,9,10,13,14,23. A prevalência no relato de um ou mais sintomas de insônia em idosos da comunidade é de $50 \%$ e não há aumento significativo com a idade 24,25,26. É maior entre as mulheres do que entre os homens $24,25,26,27$. A frequência de cochilo diurno varia de $22 \%$ a $61 \%$ dependendo do local do estudo, das características da população estudada e da definição utilizada 28 . Tanto os sintomas de insônia quanto o cochilo diurno são associados à comorbidades e ao maior risco de mortalidade em idosos 24,29,30,31.

Na literatura brasileira estão disponíveis dados sobre prevalência e fatores associados a quedas em idosos da comunidade 9,10,13,32,33. Porém, não há dados sobre a relação entre sintomas de insônia, cochilo diurno e quedas nessa população.

O presente estudo teve como objetivo analisar associações entre sintomas de insônia, cochilo diurno e ocorrência de quedas em idosos residentes na comunidade.

\section{Métodos e materiais}

Esta investigação foi realizada a partir dos dados contidos no banco eletrônico do estudo FIBRA (Rede de Estudos sobre Fragilidade em Idosos Brasileiros, pólo UNICAMP), relativos à cidade de Campinas, São Paulo, Brasil, no contexto de um estudo multicêntrico, populacional, de corte transversal, que teve como finalidade investigar a fragilidade e suas relações com variáveis sociodemográficas, psicossociais, clínicas, cognitivas, antropométricas, de capacidade funcional e de saúde física e mental em idosos da comunidade. O projeto principal foi submetido ao Comitê de Ética em Pesquisa com Seres Humanos, Faculdade de Ciências Médicas, Universidade Estadual de Campinas (UNICAMP) e aprovado mediante o parecer $n^{\circ} 208 / 2007$. O presente estudo foi submetido como adendo ao projeto principal e aprovado sob o parecer no 5302010. Foram seguidos os princípios éticos da Declaração de Helsinki e todos os participantes assinaram um Termo de Consentimento Livre e Esclarecido.

\section{Participantes}

Foi construída amostra probabilística por meio da técnica de amostragem simples dos setores censitários da zona urbana da cidade de Campinas, em cujos domicílios uma equipe treinada recrutou cotas de idosos proporcionais à sua presença por sexo e por idade na população. Os critérios de inclusão utilizados por ocasião do recrutamento foram: ter idade igual ou superior a 65 anos, ser residente permanente no domicílio e no setor censitário, ausência de comprometimentos graves de cognição, comunicação, sensoriais e mobilidade 34 . No início da sessão única de coleta de dados, foi utilizado novo critério de exclusão 34,35 , baseado no desempenho dos idosos no Mini Exame do Estado Mental (MEEM) 36,37. Dentre 900 idosos submetidos ao teste, 211 foram excluídos em virtude de não te- 
rem apresentado desempenho superior ao refletido pela nota de corte no MEEM, conforme o nível de escolaridade - 17 para os analfabetos; 22 para os idosos com escolaridade entre 1 e 4 anos; 24 para os com escolaridade entre 5 e 8 anos; e 26 para os que tinham 9 ou mais anos de escolaridade 38 . Assim, foram identificados 689 idosos sem déficit cognitivo sugestivo de demência, que foram os participantes do presente estudo. A idade média dos idosos foi de 72,1 (desvio-padrão - DP = 5,3), variando entre 65 e 90 anos estando a maioria na faixa etária de 65 a 74 anos; 68,8\% $(n=474)$ eram do sexo feminino (Tabela 1).

\section{Instrumentos e medidas}

As variáveis de interesse para esse estudo foram investigadas mediante as seguintes condições:

- Sexo e idade - duas questões de autorrelato, cujos resultados eram anotados pelos avaliadores no formulário de pesquisa: sexo (masculino ou feminino); idade dividida em quatro grupos (65 a 69,70 a 74,75 a 79, 80 e mais);

- Sintomas de insônia - construído a partir do Perfil de Saúde de Nottingham 39,40 era composto por quatro questões de autorrelato de resposta dicotômica (sim ou não) com referência aos últimos 12 meses: "O Sr(a) leva muito tempo para pegar no sono?” (dificuldade em iniciar o sono); "O Sr (a) fica acordado(a) a maior parte da noite?" (dificuldade em manter o sono); "O Sr(a) acorda de madrugada e não pega mais no sono?" (despertar precoce); “O Sr(a) dorme mal à noite?" (sono não restaurador). A resposta positiva pelo menos para uma das questões indicava a presença de sintomas de insônia 25,41.

Tabela 1

Caracterização sociodemográfica dos idosos sem déficit cognitivo sugestivo de demência segundo gênero e idade. FIBRA Campinas, São Paulo, Brasil, 2008-2009 ( $N=689$ ).

\begin{tabular}{lcc}
\hline $\begin{array}{l}\text { Variável/ } \\
\text { Categoria }\end{array}$ & Frequência & $\%$ \\
\hline Gênero & & \\
Masculino & 214 & 31,2 \\
Feminino & 474 & 68,8 \\
Idade (anos) & & \\
$65-69$ & 253 & 36,7 \\
$70-74$ & 228 & 33,1 \\
$75-79$ & 136 & 19,7 \\
$\geq 80$ & 72 & 10,5 \\
\hline
\end{tabular}

- Medicamentos para dormir - uma questão de autorrelato com resposta dicotômica (sim ou não) sobre o uso de remédios para dormir nos últimos 12 meses.

- Cochilo diurno - um item de resposta dicotômica (sim ou não) no qual era perguntado se no último ano o idoso havia cochilado, a duração diária do cochilo e sua frequência semanal 42.

- Atividades avançadas de vida diária (AAVDs) - item construído com base na literatura 43,44,45, era composto por 13 questões de autorrelato relativas a tarefas recreativas, produtivas e sociais de maior complexidade tais como, dirigir carro, dançar, viajar, fazer ou receber visitas, participar de eventos culturais (teatro, cinema, exposições), fazer trabalho remunerado, fazer trabalho voluntário, ir à igreja ou eventos sociais, participar de diretorias ou conselhos de associações (clubes, sindicatos, escolas) participar de Universidade Aberta à Terceira Idade e participar de grupos de convivência de idosos. Pelo valor da mediana, foram estabelecidos os seguintes grupos: cinco ou menos, e seis ou mais atividades avançadas de vida diária que o idoso permanecia realizando, dentre as que sempre desempenhara e dentre as que deixara de realizar.

- Atividades instrumentais de vida diária (AIVDs) - foram feitas sete questões de autorrelato que avaliaram o nível de independência para realizar o uso de transporte, fazer compras, preparar alimentos, realizar tarefas domésticas, manejo de dinheiro e uso de medicação ${ }^{46}$. Pela mediana foram estabelecidos os seguintes grupos: sete para totalmente independente nas AIVDs e seis ou menos para ajuda parcial ou total nas AIVDs.

- Atividade física - com base em itens do Minnesota Leisure Activity Questionnaire 47 validado semanticamente para o Brasil 48 , foram feitas 16 questões sobre frequência semanal e duração diária das atividades físicas realizadas pelo idoso. Seguindo-se as recomendações do American College of Sports Medicine 49 foram considerados ativos aqueles que realizassem pelo menos 150 minutos de atividade física semanal de exercícios de intensidade moderada, ou então 120 minutos de atividade física de intensidade vigorosa 50 . Os idosos que não alcançaram esses critérios foram considerados sedentários.

- Sintomas depressivos - foram avaliados por meio da Escala de Depressão Geriátrica (GDS) de 15 itens 51,52, referenciados à última semana. O ponto de corte utilizado foi de seis ou mais pontos para indicar presença de sintomas depressivos.

- Quedas - uma questão relativa à ocorrência de quedas nos últimos 12 meses, de resposta dicotômica (sim ou não). Caso o idoso respon- 
desse positivamente, era perguntado o número de ocorrências. Para os fins desta pesquisa foram consideradas as categorias: 0,1 e 2 ou mais quedas.

- Velocidade da marcha - foi cronometrado o tempo (em segundos) gasto para o idoso percorrer, em passo usual, a distância de 4,6 metros demarcados com fita adesiva. O resultado adotado foi indicado pelo tempo médio gasto para percorrer três vezes essa distância, com ajustes segundo sexo e altura. Pontuaram para baixa velocidade ou lentidão da marcha os idosos que se localizaram no extremo superior da distribuição, ou seja, que pontuaram entre os $20 \%$ que gastaram mais tempo para percorrer a distância preconizada 35 .

\section{Análise estatística}

Para descrever o perfil da amostra segundo as variáveis em estudo, foram feitas tabelas de frequência percentual das variáveis categóricas, e estatística descritiva das variáveis numéricas, com valores de média e DP. Para estudar os fatores associados com o maior número de quedas foi utilizada a análise de regressão logística univariada e multivariada, com critério stepwise de seleção de variáveis. As análises foram realizadas utilizando-se o programa computacional SAS 8.02 (SAS Inst., Cary, Estados Unidos), adotando o nível de significância de $5 \%(\mathrm{p}<0,05)$ para os testes estatísticos.

\section{Resultados}

Do total da amostra, 49,9\% queixou-se de um ou mais sintomas de insônia, com maior prevalência de despertar precoce, seguido por dificuldade em iniciar o sono, sono não restaurador e dificuldade em manter o sono. O uso de medicamentos para dormir foi relatado por $19,7 \%$ dos idosos, e a grande maioria dos participantes referiu o cochilo diurno $(62,8 \%)$. De acordo com o relato dos idosos, em média, o dispêndio de tempo semanal em cochilos diurnos no último ano foi de 166 minutos $( \pm 223)$; o número de dias na semana em que ocorriam cochilos diurnos variou de um a sete com média de 5,5 dias $( \pm 2)$ e 19,6\% dos idosos pontuaram acima da nota de corte para sintomas depressivos (GDS $\geq 6$ ). Prevaleceram os idosos classificados como sedentários (56\%). A maioria $(74,1 \%)$ mostrou-se independente funcionalmente para AIVDs e mantinham envolvimento social ou AAVDs (53,5\%). Oitenta e quatro por cento dos idosos pontuaram abaixo do valor que definia lentidão da marcha na amostra. Em relação à ocorrência de quedas no último ano, a prevalência foi de $26,2 \%$, sendo $11,9 \%$ a frequência de quedas recorrentes. Esses dados podem ser observados detalhadamente na Tabela 2 .

Como pode ser visto na Tabela 3, de acordo com a análise de regressão logística univariada, as variáveis que apresentaram associação significativa com maior número de quedas foram o sexo feminino, idade acima de 80 anos, ter hábito de tirar cochilo diurno, uso de medicamentos para dormir, sintomas depressivos, sintomas de insônia, limitação funcional em AIVDs e 90 minutos ou mais de tempo semanal acumulado em cochilos diurnos.

Com o objetivo de investigar a relação conjunta entre as variáveis que se relacionaram de maneira significativa na análise univariada, foi realizada a análise multivariada com critério stepwise de seleção de variáveis. Pelos resultados, as variáveis que apresentaram associação significativa e independente com maior número de quedas foram: sexo feminino, idade acima de 80 anos, ter hábito de tirar cochilos diurnos e sintomas depressivos (Tabela 4).

\section{Discussão}

A prevalência no relato de um ou mais sintomas de insônia foi de 49,9\%, dado compatível com o de pesquisas internacionais e nacionais. Por exemplo, no estudo multicêntrico Established Populations for Epidemiologic Studies of the Elderly (EPESE), envolvendo 9.282 idosos da comunidade, cerca de $40 \%$ dos entrevistados relataram dificuldade em iniciar ou manter o sono 24 . No levantamento realizado pelo $\mathrm{Na}$ tional Sleep Foundation, 46\% dos idosos entre 65 e 74 anos e 50\% daqueles entre 75 e 84 anos relataram pelo menos uma queixa relativa ao sono noturno 53. Em estudos desenvolvidos no Brasil a prevalência foi de $38,9 \% 54$ e $42 \% 55$. Em relação ao cochilo diurno, 62,9\% dos entrevistados responderam positivamente, cochilando em média cinco dias por semana com duração diária de 48,1 minutos. Em pesquisa realizada nos Estados Unidos, cerca de $40 \%$ dos idosos entrevistados relataram cochilar de quatro a sete vezes por semana, enquanto $46 \%$ o faziam de uma a três vezes 53 . Entre idosos chineses, a prevalência do cochilo foi de $50,4 \%$, de três a quatro vezes por semana, e 35,4\% diariamente 27 . Segundo os estudiosos, as pesquisas epidemiológicas que avaliam a frequência de sintomas de insônia e cochilo diurno na população idosa sofrem variação na prevalência, principalmente em decorrência dos critérios metodológicos de análise, bem como do contexto cultural e da população envolvida 25,28 . 
Caracterização dos idosos conforme sintomas de insônia, consumo de medicamentos para dormir, cochilo diurno, atividade física, capacidade funcional, velocidade da marcha, sintomas depressivos e quedas. FlBRA Campinas, São Paulo, Brasil, 2008-2009 ( $N=689$ ).

\begin{tabular}{|c|c|c|c|c|}
\hline Variável & $\mathrm{n}$ & Categoria & Média \pm DP & $\begin{array}{c}\text { Frequência } \\
\text { (\%) }\end{array}$ \\
\hline \multirow[t]{2}{*}{ Sintomas de insônia } & 679 & Sim & & 49,9 \\
\hline & & Não & & 50,1 \\
\hline \multirow[t]{2}{*}{ Dificuldade em iniciar o sono } & 678 & Sim & & 28,3 \\
\hline & & Não & & 71,7 \\
\hline \multirow[t]{2}{*}{ Dificuldade em manter o sono } & 678 & Sim & & 20,8 \\
\hline & & Não & & 79,2 \\
\hline \multirow[t]{2}{*}{ Despertar precoce } & 678 & Sim & & 34,5 \\
\hline & & Não & & 65,5 \\
\hline \multirow[t]{2}{*}{ Sono não restaurador } & 677 & Sim & & 28,2 \\
\hline & & Não & & 71,8 \\
\hline \multirow[t]{2}{*}{ Medicamentos para dormir } & 675 & Sim & & 19,7 \\
\hline & & Não & & 80,3 \\
\hline \multirow[t]{2}{*}{ Cochilo diurno nos últimos 12 meses } & 688 & Sim & & 62,8 \\
\hline & & Não & & 37,2 \\
\hline Frequência semanal do cochilo & 424 & & $5,5 \pm 3,0$ & \\
\hline Duração diária do cochilo; minutos & 421 & & $48,1 \pm 35,5$ & \\
\hline Duração semanal do cochilo; minutos & 674 & & $166,7 \pm 223,0$ & \\
\hline \multirow[t]{2}{*}{ Atividade física (minutos) } & 688 & Ativo * & & 43,3 \\
\hline & & Sedentário & & 56,7 \\
\hline Duração semanal em atividades moderadas (minutos) & 688 & & $173,7 \pm 250,5$ & \\
\hline Duração diária em atividades moderadas (minutos) & 688 & & $50,4 \pm 64,0$ & \\
\hline Duração semanal em atividades vigorosas (minutos) & 688 & & $10,9 \pm 78,1$ & \\
\hline Duração diária em atividades vigoras (minutos) & 688 & & $3,7 \pm 24,4$ & \\
\hline \multirow[t]{2}{*}{ AAVDs } & 665 & $\leq 5$ & & 46,5 \\
\hline & & $\geq 6$ & & 53,5 \\
\hline Número de AAVDs que ainda faz & 665 & & $5,6 \pm 2,2$ & \\
\hline \multirow[t]{2}{*}{ AlVDs } & 668 & $\leq 6$ & & 25,9 \\
\hline & & 7 & & 74,1 \\
\hline Número de AIVDs independentes & 668 & & $6,5 \pm 1,0$ & \\
\hline \multirow[t]{2}{*}{ Velocidade da marcha } & 687 & Não frágil & & 84,1 \\
\hline & & Frágil & & 15,9 \\
\hline Tempo gasto no teste de velocidade da marcha, segundos & 687 & & $5,0 \pm 1,3$ & \\
\hline \multirow[t]{2}{*}{ Sintomas depressivos } & 673 & Sim & & 19,6 \\
\hline & & Não & & 80,4 \\
\hline Escore GDS & 673 & & $3,5 \pm 2,8$ & \\
\hline \multirow[t]{3}{*}{ Quedas } & 635 & 0 & & 73,7 \\
\hline & & 1 & & 14,4 \\
\hline & & $\geq 2$ & & 11,9 \\
\hline Número de quedas & 635 & & $0,4 \pm 0,7$ & \\
\hline
\end{tabular}

AAVDs: atividades avançadas de vida diária; AIVDs: atividades instrumentais de vida diária; DP: desvio-padrão; GDS: Escala de Depressão Geriátrica.

* $\geq 150$ minutos de atividade moderada ou 120 minutos de atividade vigorosa por semana.

Nota: as diferenças apresentadas em relação ao número da amostra referem-se à ausência de respostas no protocolo da pesquisa. 
Resultados da análise de regressão logística univariada para número de quedas. FIBRA Campinas, São Paulo, Brasil, 2008$2009(n=635)$

\begin{tabular}{|c|c|c|c|}
\hline Variável/Categorias & Valor de $p$ & OR * bruta & $1 \mathrm{C95 \%}$ \\
\hline \multicolumn{4}{|l|}{ Sexo } \\
\hline Masculino (referência) & & 1,00 & \\
\hline Feminino & $<0,001$ & 6,25 & $2,67-14,65$ \\
\hline \multicolumn{4}{|l|}{ Idade (anos) } \\
\hline 65-69 (referência) & & 1,00 & \\
\hline $70-74$ & 0,356 & 1,34 & $0,72-2,46$ \\
\hline $75-79$ & 0,248 & 1,50 & $0,75-3,00$ \\
\hline$\geq 80$ & 0,004 & 2,96 & $1,40-6,23$ \\
\hline \multicolumn{4}{|l|}{ Atividade física } \\
\hline Ativo ** (referência) & & 1,00 & \\
\hline Sedentário & 0,954 & 0,99 & $0,61-1,60$ \\
\hline \multicolumn{4}{|l|}{ Velocidade da marcha } \\
\hline Não-frágil (referência) & & 1,00 & \\
\hline Frágil & 0,276 & 1,41 & $0,76-2,59$ \\
\hline \multicolumn{4}{|l|}{ AAVDs } \\
\hline$\geq 6$ (referência) & & 1,00 & - \\
\hline$\leq 5$ & 0,13 & 1,45 & $0,90-2,36$ \\
\hline \multicolumn{4}{|l|}{ AIVDs } \\
\hline$\geq 7$ (referência) & & 1,00 & - \\
\hline$\leq 6$ & 0,031 & 1,76 & $1,05-2,93$ \\
\hline \multicolumn{4}{|l|}{ Medicamentos para dormir } \\
\hline Não (referência) & & 1,00 & \\
\hline Sim & 0,01 & 2,02 & $1,18-3,46$ \\
\hline \multicolumn{4}{|l|}{ Sintomas de insônia } \\
\hline Não (referência) & & 1,00 & \\
\hline Sim & 0,018 & 1,82 & $1,11-2,99$ \\
\hline \multicolumn{4}{|l|}{ Cochilos diurnos } \\
\hline Não (referência) & & 1,00 & \\
\hline Sim & 0,007 & 2,18 & $1,24-3,84$ \\
\hline \multicolumn{4}{|c|}{ Duração semanal do cochilo diurno (minutos) } \\
\hline < 90 (referência) & & 1,00 & \\
\hline$\geq 90$ & 0,029 & 1,74 & $1,06-2,87$ \\
\hline \multicolumn{4}{|l|}{ Sintomas depressivos } \\
\hline Não (referência) & & 1,00 & \\
\hline Sim & 0,011 & 2,01 & $1,17-3,43$ \\
\hline
\end{tabular}

AAVDs: atividades avançadas de vida diária; AIVDs: atividades instrumentais de vida diária; IC95\%: intervalo de 95\% de confiança; OR: odds ratio.

* Razão de risco para mais quedas ( $n=559$ para 0-1 queda e $n=76$ para 2 ou mais quedas);

** $\geq 150$ minutos de atividade moderada ou 120 minutos de atividade vigorosa por semana.

Quanto a prevalência de quedas no último ano, os dados deste estudo replicam os de pesquisas anteriores realizadas no Brasil 9,10,13 e em outros países da América Latina e Caribe 8. Do mesmo modo como vem sendo apontado por outros estudos, tanto o sexo feminino quanto a idade apresentaram associação significativa com o maior número de quedas 7,8,910,11,12,13. Em recente metanálise, os resultados mostraram que o incremento de cinco anos na idade aumentou o risco de cair em 1,12 vezes tanto para um evento quanto para duas ou mais quedas em idosos da comunidade 7. Possivelmente, fatores tais como maior variabilidade da marcha, maior 
Resultados da análise de regressão logística multivariada para número de quedas. FIBRA Campinas, São Paulo, Brasil, 2008$2009(n=594)$.

\begin{tabular}{|c|c|c|c|}
\hline Variável/Categorias & Valor de $p$ & OR * ajustada & IC95\% \\
\hline \multicolumn{4}{|l|}{ Sexo } \\
\hline Masculino (referência) & & 1,00 & \\
\hline Feminino & $<0,001$ & 7,73 & $3,03-19,72$ \\
\hline \multicolumn{4}{|l|}{ Idade (anos) } \\
\hline 65-69 (referência) & & 1,00 & \\
\hline $70-74$ & 0,145 & 1,63 & $0,84-3,16$ \\
\hline $75-79$ & 0,366 & 1,42 & $0,66-3,04$ \\
\hline$\geq 80$ & 0,003 & 3,48 & $1,54-7,85$ \\
\hline \multicolumn{4}{|l|}{ Cochilo diurno } \\
\hline Não (referência) & & 1,00 & \\
\hline Sim & 0,008 & 2,24 & $1,24-4,05$ \\
\hline \multicolumn{4}{|l|}{ Sintomas depressivos } \\
\hline Não (referência) & & 1,00 & \\
\hline Sim & 0,021 & 1,98 & $1,11-3,53$ \\
\hline
\end{tabular}

IC95\%: intervalo de 95\% de confiança; OR: odds ratio.

* Razão de risco para mais quedas ( $n=523$ para 0-1 queda e $n=71$ para 2 ou mais quedas). Nota: critério stepwise de seleção de variáveis.

fragilidade e incapacidade funcional, bem como maior número de doenças crônicas podem estar envolvidos com a ocorrência de quedas recorrentes nesses grupos 7,13,35,56,57,58. Para as mulheres, a maior frequência de queixas relacionadas ao sono, principalmente na presença de comorbidades, relaciona-se com quedas 11,13,54,59. Quanto à idade, há uma tendência dos idosos acima dos 80 anos dormirem menos ou mais tempo durante a noite, quando comparados aos idosos mais jovens 26,54 . Contudo, a qualidade do sono de idosos acima de 80 anos não difere de modo significativo dos idosos mais jovens e pode até ser melhor quando a relação é controlada por status socioeconômico, suporte social e familiar, bem como por condições de saúde 26,59. Piores condições de saúde e fatores psicossociais influenciam a relação entre sintomas de insônia, cochilo diurno e quedas em idosos mais velhos 11,13,26,29,58,60.

Neste estudo foi observada associação significativa entre sintomas depressivos e maior número de quedas. Existiriam pelo menos três explicações para a relação entre quedas e depressão: a depressão pode preceder as quedas e vice versa, assim como ambas podem ser efeito de uma causa comum tais como pior condição de saúde, limitação funcional em atividades de vida diária, declínio cognitivo e baixa velocidade da marcha. Dentre as variáveis sociodemográficas, o sexo feminino é um fator de risco robusto para quedas e depressão em idosos da comunidade 7,23,61. Além disso, tem sido observado que o cochilo diurno é fator associado à depressão em idosos 60 .

Embora tenha havido associação entre sintomas de insônia e quedas na análise univariada, essa relação não se manteve no modelo ajustado. Diferentemente, em estudo prospectivo envolvendo 622 idosos da comunidade, com o objetivo de avaliar a prevalência de quedas e os fatores associados, os problemas do sono foram apontados como um dos fatores de risco de quedas nesse grupo 11. Com objetivos semelhantes, outro estudo envolvendo 2.096 idosos mostrou que os participantes que relataram problemas relacionados ao sono apresentaram probabilidade aumentada de duas ou mais quedas quando comparados aos que não relataram ${ }^{12}$. No estudo multicêntrico Study of Osteoporotic Fractures (SOF) o grupo de pesquisadores observou que mulheres que dormiam menos de 5 horas por noite apresentaram risco aumentado de quedas recorrentes quando comparadas as que dormiam entre 7 e 8 horas, porém essa relação foi atenuada após ajuste multivariado. Também foi encontrado que a eficiência do sono abaixo de $70 \%$ e despertares repetitivos com duração total acima de 120 minutos por noite relacionaram-se com risco para duas ou mais quedas independente da 
idade, raça, consumo de psicoativos, índice de massa corporal (IMC), depressão, exercício físico, AIVDs e comorbidades. Não foi encontrada associação significativa entre cochilo diurno e quedas, nem tampouco entre cochilo e tempo total de sono noturno. Ainda de acordo com os resultados, em geral, a magnitude das associações entre as medidas do sono e o risco de quedas foi reduzida pela adição da variável velocidade da marcha nos modelos de análise, sugerindo que a piora na qualidade do desempenho físico pode explicar em parte a associação entre o sono e risco para quedas 20 .

No estudo envolvendo 1.542 idosos da comunidade com média de idade de 77,4 anos, houve maior prevalência de uso de medicamentos para dormir, dificuldade de iniciar o sono, fragmentação do sono, despertar precoce e sonolência diurna entre os idosos que sofreram quedas recorrentes. Quando comparados aos que dormiam entre 7 e 8 horas por noite, aqueles que dormiam acima de 11 horas foram mais prevalentes em quedas recorrentes. Dormir acima de 11 horas esteve associado com maior risco de sofrer uma ou mais quedas em indivíduos acima de 75 anos e nas mulheres, mesmo após controle por fatores de confusão. Também foi encontrada associação entre dormir menos de 5 horas por noite e uma ou mais quedas nos idosos acima de 75 anos 62. Em outro estudo transversal cujo objetivo foi avaliar a associação entre gênero, horas de sono noturno e quedas em idosos da comunidade, aqueles que relataram dormir menos que 5 horas apresentaram maior risco de quedas quando comparados aos que dormiam entre 5 e 7,9 horas por noite. No entanto, essa relação só foi significativa entre as mulheres 22 . A análise da relação entre o autorrelato de queixas do sono e incontinência urinária e o risco de quedas em mulheres acima de 70 anos, resultou em uma chance duas vezes maior de quedas entre aquelas que apresentavam sonolência diurna do que nas idosas sem esse sintoma, mesmo após o controle de fatores de risco associados 17. Em idosos com diagnóstico de apneia do sono foi observado maior risco de quedas do que para os idosos sem apneia do sono 19 .

Ainda no estudo SOF, os pesquisadores investigaram a relação entre hábitos de sono e cochilo diurno autorrelatados e a ocorrência de quedas e fraturas. Após ajuste multivariado, os resultados mostraram que as mulheres que afirmaram cochilar apresentaram maior risco para duas ou mais quedas e fratura de quadril do que as que não relataram cochilar. Quanto à duração do cochilo, aquelas que cochilavam pelo menos 3 horas por semana tiveram maior probabilidade de duas ou mais quedas que as que cochila- vam menos que 3 horas. A inclusão de força do quadríceps no modelo multivariado não afetou a relação entre cochilo diurno e queda. Independentemente do estado de depressão, mulheres que cochilavam de dia apresentaram risco de sofrer duas ou mais quedas no seguimento quando comparadas as que não relataram cochilar 18. No estudo envolvendo 572 idosos da comunidade, foi observado que os participantes que relataram cochilar mais do que 30 minutos por dia tinham três vezes mais chances de cair, controlados os demais fatores de risco de quedas 21 .

Os mecanismos fisiológicos envolvidos na relação entre sintomas de insônia, cochilo diurno e quedas em idosos da comunidade ainda são pouco esclarecidos. Possivelmente são multifatoriais e incluem a diminuição do equilíbrio e do tempo de reação postural, e o efeito residual dos medicamentos usados para tratar os problemas do sono 20,63. O estudo multicêntrico Osteoporotic Fractures in Men Study (MrOS), avaliou 2.862 homens acima de 67 anos por meio de medidas objetivas e subjetivas de sono. Após ajuste multivariado, os resultados indicaram que a latência do sono acima ou igual a 30 minutos, despertares noturnos acima de 90 minutos e ter menos de $80 \%$ de eficiência do sono estiveram associados a menor força de preensão, menor velocidade da marcha, incapacidade de levantar-se de uma cadeira sem assistência, e inabilidade em percorrer pequenos trajetos 63 . Nessa mesma linha, pesquisadores encontraram associação entre cochilo diurno e menor velocidade da marcha 64. É possível afirmar que a fragmentação do sono noturno é menos restauradora do que uma noite de sono sem sucessivos despertares podendo acarretar pior desempenho funcional diurno. De todo modo, esse conjunto de dados não autoriza que se apontem relações simples e unidirecionais de causalidade entre estas variáveis e problemas de sono, nem entre estes e o aumento do risco para quedas em idosos. $\mathrm{O}$ que se pode afirmar é que problemas de sono e quedas são fenômenos complexos e multideterminados, que interagem com variáveis de saúde e contextuais.

Este estudo revelou associações ainda pouco exploradas na literatura gerontológica, de modo especial a brasileira, na qual não há estudos semelhantes. Embora seja de natureza transversal, o fato de ter envolvido amostra probabilística de uma cidade de médio porte, com bons índices de desenvolvimento econômico, significa uma contribuição importante à compreensão do fenômeno quedas em idosos no Brasil.

Entender a contribuição relativa dos fatores de risco de quedas em idosos permite que os profissionais de saúde possam elaborar medidas de 
intervenção apropriadas. As quedas constituem uma síndrome geriátrica, e caracterizá-la pressupõe a integração e a contribuição de múltiplos fatores. A estratégia potencialmente mais eficaz para a redução das taxas de prevalência de quedas em idosos da comunidade é intervir em fato- res de risco modificáveis 65 , entre eles o sono noturno e o cochilo diurno. Futuras pesquisas poderão investigar o impacto de intervenções dessa natureza sobre os problemas do sono e sobre o cochilo, como variáveis relacionadas à ocorrência de quedas em idosos da comunidade.

\section{Resumen}

Esta investigación se centró en las asociaciones entre síntomas de insomnio, siesta breve diurna, y caídas en ancianos de una comunidad desfavorecida. Estudio transversal de base poblacional y muestreo probabilístico involucrando a 689 ancianos de una comunidad desfavorecida. El protocolo contenía variables de autorrelato y de desempeño físico. Se utilizó un análisis de regresión logística univariada y multivariada, y el nivel de significancia estadística adoptado fue de $p<$ 0,05 . La prevalencia de síntomas de insomnio y siesta breve diurna fue de 49,9\% ( $n=339)$ y 62,8\% $(n=432)$, respectivamente. Catorce coma cuatro por ciento relataron una única caída y 11,9\% relataron caídas re- currentes. La ocurrencia de caídas se asoció con el sexo femenino (OR = 7,73; IC95\%: 3,03-19,72), edad $\geq 80$ años (OR =3,48; IC95\%: 1,54-7,85), siesta breve diurna $(O R=2,24$; IC95\%: 1,24-4,05) y sintomas depresivos $(O R=1,98 ;$ IC95\%: 1,11-3,53). La asociación entre siesta breve diurna y caídas replican datos de investigaciones internacionales. Identificar factores de riesgo modificables pueden auxiliar en programas de prevención de caídas.

Insomnio; Privación del Sueño; Trastornos del Sueño; Accidentes por Caídas; Anciano 


\section{Agradecimentos}

Ao Conselho Nacional de Desenvolvimento Científico e Tecnológico (CNPq, processo no 555082- 2006-7) pelo financiamento do Estudo FIBRA (Rede de Estudos sobre Fragilidade em Idosos Brasileiros) pólo Universidade Estadual de Campinas (UNICAMP). À Coordenação de Aperfeiçoamento de Pessoal de Nível Superior (CAPES) pela bolsa de mestrado de A. A. Pereira.

\section{Referências}

1. Fabrício SCC, Rodrigues RAP, Costa Junior ML. Causas e consequências de quedas de idosos atendidos em hospital público. Rev Saúde Pública 2004; 38:93-9.

2. Kannus P, Sievanen H, Palvanen M, Jarvinen T, Parkkari J. Prevention of falls and consequent injuries in elderly people. Lancet 2005; 366:1885-93.

3. Ribeiro AP, Souza ER, Atie S, Souza AC, Schilithz AO. A influência das quedas na qualidade de vida de idosos. Ciênc Saúde Coletiva 2008; 13:1265-73.

4. Rubenstein LZ. Falls in older people: epidemiology, risk factors and strategies for prevention. Age Ageing 2006; 35 Suppl 2:ii37-41.

5. Bohl AA, Fishman PA, Ciol MA, Williams B, Logerfo J, Phelan EA. A longitudinal analysis of total 3-year heath care costs for older adults who experience a fall requiring medical care. J Am Geriatr Soc 2010; 58:853-60.

6. Tinetti ME, Kumar C. The patient who falls. JAMA 2010; 303:258-66.

7. Deandrea S, Lucenteforte E, Bravi F, Foschi R, La Vecchia C, Negri E. Risk factors for falls in community-dwelling older people: a systematic review and meta-analysis. Epidemiology 2010; 21:658-68.

8. Reyes-Ortiz CA, Al Snih S, Markides KS. Falls among elderly persons in Latin America and the Caribbean and among elderly Mexican-Americans. Rev Panam Salud Pública 2005; 17:362-9.

\section{Colaboradores}

A. A. Pereira, M. F. Ceolim e A. L. Neri foram responsáveis pelo desenho do estudo, análise dos dados e pela elaboração do texto do artigo.
9. Siqueira FV, Facchini LA, Piccini RX, Tomasi E, Thumé E, Silveira DS, et al. Prevalência de quedas em idosos e fatores associados. Rev Saúde Pública 2007; 41:749-56.

10. Siqueira FV, Facchini LA, Silveira DS, Piccini RX, Tomasi E, Thumé E, et al. Prevalence of falls in elderly in Brazil: a countrywide analysis. Cad Saúde Pública 2011; 27:1819-26.

11. Gabmann KG, Rupprecht R, Freiberger E. Predictors for occasional and recurrent falls in community-dwelling older people. Z Gerontol Geriat 2009; 42:3-10.

12. Bekibele CO, Gureje O. Fall incidence in a population of elderly persons in Nigeria. Gerontology 2010; 56:278-83.

13. Coimbra AMV, Ricci NA, Coimbra IB, Costallat LTL. Falls in the elderly of the family health program. Arch Gerontol Geriatr 2010; 51:317-22.

14. Bloch F, Thibaud M, Dugué B, Brèque C, Rigaud AS, Kemoun G. Psychotropic drugs and falls in the elderly people: update literature review and metaanalysis. J Aging Health 2011; 23:329-46.

15. Syllias H, Idland G, Sandvik L, Forsen L, Bergland A. Does mortality of the aged increase with the number of falls? Results from a nine-year followup study. Eur J Epidemiol 2011; 24:351-5. 
16. Alamgir H, Muazzan S, Nasrullah M. Unintentional falls mortality among elderly in the United States: time for action. Injury 2012; 43:2065-71.

17. Teo JSH, Briffa NK, Devine A, Dhaliwal SS, Prince RL. Do sleep problems or urinary incontinence predict falls in elderly women? Aust J Physiother 2006; 52:19-24.

18. Stone KL, Ewing SK, Lui LY, Ensrud KE, AncoliIsrael S, Bauer DC, et al. Self-reported sleep and nap habits and risk of falls and fractures in older women: the study of osteoporotic fractures. J Am Geriatr Soc 2006; 54:1177-83.

19. Kaushik S, Wang JJ, Mitchell P. Sleep apnea and falls in older people. J Am Geriatr Soc 2007; 55:1149-50.

20. Stone KL, Ancoli-Israel S, Blackwell T, Ensrud KE, Cauley JA, Redline S, et al. Actigraphy-measured sleep characteristics and risk of falls in older women. Arch Inter Med 2008; 168:1768-75.

21. George RJS, Delbaere K, Williams P, Lord S. Sleep quality and falls in older people living in self and assisted-care villages. Gerontology 2009; 55:162-8.

22. Hsuo-Ko K, Yang CCH, Yu YH, Tsai KT, Chen CY. Gender-Specific association between self-reported sleep duration in high-functioning older adults. J Gerontol A Biol Sci Med Sci 2010; 65:190-6.

23. Biderman A, Cwikel J, Fried AV, Galinsk D. Depression and falls among community dwelling elderly people: a search for common risk factors. J Epidemiol Community Health 2002; 56: 631-6.

24. Foley DJ, Monjan AA, Brown SL, Simonsick EM, Wallace RB, Blazer DG. Sleep complaints among elderly persons: an epidemiologic study of three communities. Sleep 1995; 18:425-32.

25. Ohayon MM. Epidemiology of insomnia: what we know and what we still need to learn. Sleep Med Rev 2002; 6:97-111.

26. Gu D, Sautter J, Pipkin R, ZengY. Sociodemographic and health correlates of sleep quality and duration among very old Chinese. Sleep 2010; 33:601-10.

27. Xianchen L, Lianqui L. Sleep habits and insomnia in a sample of elderly persons in China. Sleep 2005; 28:1579-87.

28. Martin JL, Ancoli-Israel S. Napping in older adults. Sleep Med Clin 2006; 1:177-86.

29. Foley DJ, Ancoli-Israel S, Britz P, Walsh J. Sleep disturbance and chronic disease in older adults: results of the 2003 National Sleep Foundation Sleep in American Survey. J Psychosom Res 2004; 56:497-502.

30. Stone KL, Ewing SK, Ancoli-Israel S, Ensrud KE, Redline S, Bauer DC, et al. Self-reported sleep and nap habits and risk of mortality in large cohort of older women. J Am Geriatr Soc 2009; 57:604-11.

31. Castro-Costa E, Dewey ME, Ferri CP, Uchôa E, Firmo JO, Rocha FL, et al. Association between sleep duration and all-cause mortality in old age: 9 year follow-up of the Bambuí Cohort Study, Brazil. J Sleep Res 2011; 20:303-10.

32. Perracine MR, Ramos LR. Fatores associados a quedas em uma coorte de idosos residentes na comunidade. Rev Saúde Pública 2002; 36:709-16.

33. Cruz DT, Ribeiro LC, Vieira MT, Teixeira MTB, Bastos RR, Leite ICG. Prevalência de quedas e fatores associados em idosos. Rev Saúde Pública 2012; 46:138-46.
34. Ferrucci L, Guralnik JM, Studenski S, Fried LP, Culter Jr. GB, Waltson JD, et al. Designing randomized, controlled trials aimed at preventing or delaying functional decline and disability in frail, older persons: a consensus report. J Am Geriatr Soc 2004; 52:625-34

35. Fried LP, Tangen CM, Walston J, Newman AB, Hirsch C, Gottdiener J, et al. Frailty in older adults: evidence for a phenotype. J Gerontol A Biol Sci Med Sci 2001; 56:M146-56.

36. Folstein MF, Folstein SE, McHugh PR. "Mini-mental state". A practical method for grading the cognitive state of patients for the clinician. J Psychiatr Res 1975; 12:189-98.

37. Bertolucci PHF, Brucki SMD, Campacci S, Juliano Y. O Mini-Exame do Estado Mental em uma população geral: impacto da escolaridade. Arq Neuropsiquiatr 1994; 52:1-7.

38. Brucki SM, Nitrini R, Caramelli P, Bertolucci PH, Okamoto IH. Sugestões para o uso do Mini-exame do Estado Mental no Brasil. Arq Neuropsiquiatr 2003; 61:777-81.

39. Hunt SM, McKenna SP, McEwen EM, Backett JW, Papp E. A quantitative approach to perceived health status: a validation study. J Epidemiol Community Health 1980; 34:281-6.

40. Teixeira-Salmela LF, Magalhães LC, Souza AC, Lima MC, Lima LCM, Goulart F. Adaptação do Perfil de Saúde de Nottingham: um instrumento simples de avaliação da qualidade de vida. Cad Saúde Pública 2004; 20:905-14.

41. Edinger JD, Bonnet MH, Bootzin RR, Doghramji K, Dorsey CM, Espie CA, et al. Derivation of research diagnostic criteria for insomnia: report of an American Academy of Sleep Medicine work group. Sleep 2004; 27:1567-96.

42. Klumb PL, Baltes MM. Validity of retrospective time-use reports in old age. Appl Cognit Psychol 1999; 13:527-39.

43. Reuben DB, Laliberte L, Hiris J, Mor V. A hierarchical exercise scale to measure function at the Advanced Activities of Daily Living (AADL) level. J Am Geriatr Soc 1990; 38:855-61.

44. Baltes MM, Mayr U, Borchelt M, Maas I, Wilms HU. Everyday competence in old and very old age: an interdisciplinary perspective. Ageing Soc 1993; 13:657-80.

45. StrawbridgeWJ, Wallhagen MI, Cohen RD. Successful aging and well-being: self-rated compared with Rowe and Kahn. Gerontologist 2002; 42:727-33.

46. Lawton M, Brody E. Assessment of older people: self-maintaining and instrumental activities of daily living. Gerontologist 1969; 9:179-86.

47. Taylor HL, Jacobs Jr. DR, Schucker B, Knudsen J, Leon AS, Debacker G. A questionnaire for the assessment of leisure time physical activities. J Chronic Dis 1978; 31:741-55.

48. Lustosa LP, Pereira DS, Dias RC. Tradução e adaptação transcultural do Minnesota Leisure Activities Questionnaire em idosos. Geriatria \& Gerontologia 2011; 5:57-65. 
49. Nelson ME, Jack R, Blair SN, Duncan PW, Judge JO, King AC, et al. Physical activity and public health in older adults: recommendation from the American College of Sports Medicine and the American Heart Association. Med Sci Sports Exerc 2007; 39:1435-45.

50. Haskell WL, I-Min L, Pate RR, Powell KE, Blair SN, Frnaklin BA, et al. Physical activity and public health: updated recommendation for adults from the American College of Sports Medicine and the American Heart Association. Med Sci Sports Exerc 2007; 39:1423-34.

51. Yesavage JA, Brink TL, Rose TL, Lum O, Huang V, Adey M, et al. Development and validation of a geriatric depression screening scale: a preliminary report. J Psychiatr Res 1983; 17:37-49.

52. Almeida OP, Almeida SA. Short versions of the Geriatric Depression Scale: A study of their validity for the diagnosis of major depressive episode according to ICD-10 and DSM-IV. Int J Geriat Psychiatry 1999; 14:858-65.

53. National Sleep Foundation. Sleep in America poll. http://www.sleepfoundation.org/polls/200 3SleepPollExecutiveSumm.pdf (acessado em 14/ Mar/2012).

54. Rocha FL, Uchôa E, Guerra HL, Firmo JO, Vidigal PG, Lima-Costa MF. Prevalence of sleep complaints and associated factors in community-dwelling older people in Brazil: the Bambuí Health and Ageing Study (BHAS). Sleep Med 2002; 3:231-8.

55. Bittencourt LRA, Santos-Silva R, Taddei JA, Andersen ML, Mello MT, Tufik S. Sleep complaints in the adult Brazilian population: a national survey based screening questions. J Clin Sleep Med 2009; 5:459-63.

56. Callisaya ML, Blizzard L, Schmidt MD, Martin KL, McGinley JL, Sanders LM, et al. Gait, gait variability and the risk of multiple incidence falls in older people: a population-based study. Age Ageing 2011; 40:481-7.

57. Camargos MCS, Perpétuo IHO, Machado CJ. Life expectancy with functional disability in elderly persons in São Paulo, Brazil. Rev Panam Salud Pública 2005; 17:379-86.
58. Marengoni A, Angleman S, Melis R, Mangialasche F, Karp A, Garmen A, et al. Aging with multimorbidity: a systematic review of the literature. Ageing Res Rev 2011; 10:430-9.

59. Blay SL, Andreoli SB, Gastal FL. Prevalence of selfreported sleep disturbance among older adults and the association of disturbed sleep with service demand and medical conditions. Int Psychogeriatr 2008; 20:582-95.

60. Foley DJ, Vitiello MV, Bliwise DL, Ancoli-Iarael S, Monjan AA, Walsh JK. Frequent napping is associated with excessive daytime sleepiness, depression, pain, and nocturia in older adults: findings from the National Sleep Foundation "2003 Sleep in American Poll”. Am J Geriatr Psychiatry 2007; 15:344-50.

61. Cole MG, Dendukuri N. Risk factors for depression among elderly community subjects: a systematic review and meta-analysis. Am J Psychiatry 2003; 160:1147-56.

62. Mesas AE, López-García E, Rodríguez-Artanejo F. Self-reported sleep duration and falls in older adults. J. Sleep Res 2011; 20:21-7.

63. Dam TTL, Ewing S, Ancoli-Israel S, Ensrud K, Redline S, Stone K. Association between sleep and physical function in older men: The Osteoporotic Fractures in Men Sleep Study. J Am Geriatr Soc 2008; 56:1665-73.

64. Goldman SE, Stone KL, Ancoli-Israel S, Blackwell T, Ewing SK, Boudreau R, et al. Poor sleep is associated with poorer physical performance and greater functional limitations in older women. Sleep 2007; 30:1317-24.

65. Gillespie LD, Robertson MC, Gillespie WJ. Interventions for preventing falls in elderly people. Cochrane Database of Syst Rev 2009; (15):CD007146.

Recebido em 16/Jul/2012

Versão final reapresentada em 23/Out/2012 Aprovado em 13/Nov/2012 